\title{
Una continuación dieciochesca del Quijote: la Historia del más famoso escudero Sancho Panza
}

\author{
Norberto PÉrez García*
}

Es historia sabida. Paralela al éxito editorial y a la riqueza variadísima de lecturas del Quijote discurre la corriente agitada de imitaciones, parodias, remedos, paráfrasis o continuaciones de la obra mayor de Cervantes: desde el propio Quijote apócrifo de Avellaneda hasta el reciente Al morir don Quijote de Andrés Trapiello la lista es casi inabarcable.

El siglo XVIII, que marca el punto de inicio de la consideración canónica de la novela, no podía ser ajeno a estos hechos, y las ediciones de las obras cervantinas, particularmente del Quijote, y los interesantes estudios críticos publicados se vieron acompañados de continuaciones e imitaciones en diversos países y, claro está, en España ${ }^{1}$.

Al lado de la figura del hidalgo, y especialmente a finales de este siglo, empieza a cobrar protagonismo en las recreaciones cervantinas el personaje de Sancho Panza ${ }^{2}$. En 1786 Jacinto María Delgado publicaba unas Adiciones a la historia del Ingenioso hidalgo don Quijote de la Mancha en que se prosiguen los sucesos ocurridos a su escudero Sancho Panza que pronto serían acompañadas por la Historia del más famoso escudero Sancho Panza, desde la gloriosa muerte de don Quijote hasta el último día y postrera hora de su vida.

Esta novela apareció publicada en dos partes, en 1793 y 1798 respectivamente, y fue escrita, posiblemente, por dos creadores distintos, aunque en ninguno de estos libros aparece el nombre de autor.

* I.E.S. La Poveda, Arganda (Madrid).

1. Una primera aproximación al tema en Aguilar Piñal, Francisco, "Cervantes en el siglo XVIII", Anales Cervantinos, XXI, 1983, pp. 153-163.

2. Cfr. Flores, Robert M., Sancho Panza through three hundred seventy-five years of continuations, imitations and criticism, 1605-1980, Newark, Delaware, Juan de la Cuesta, 1982. 
La Primera Parte es, indudablemente, obra del Bachiller Pedro Gatell, tal y como demuestran las alusiones que deslizó su autor en otras obras de temática cervantina aparecidas en años anteriores, y se publicó, póstuma, en 1793 en Madrid, por la imprenta Real. La Segunda Parte, impresa en Madrid en 1798 por la imprenta Villalpando, sería obra de un autor anónimo que decide continuar la incompleta historia de Gatell, como indica en el prólogo ${ }^{3}$ :

El autor de la Primera Parte de esta obrita, hace cuatro años que murió, y aunque ofreció continuarla con el segundo tomo, como en su primero verás, en el escrutinio de sus papeles, por más cuidado que se ha puesto, no se han hallado ni originales ni borradores que traten de esto: por este accidente, quedó imperfecta la obra, y sin gloria su autor, pues como los adictos a saber, ven solo su primer tomo, la desprecian, aunque el mérito sea sublime, porque asunto que se propone, y no se concluye mal o bien, poco merece, por esto dispuse (con el fin de que el primer tomo luciese) formar este segundo, siguiendo la idea del primero, dándole conclusión con la muerte de Sancho, como en él previene. (Segunda Parte, sin numerar, folios 2 y 3$)^{4}$.

Sea como fuere, el primer autor, Pedro Pablo Gatell y Carnicer, cuya figura empieza a conocerse gracias a los trabajos de Elisabel Larriba ${ }^{5}$, fue un intelectual ilustrado que en el ocaso de su vida abandonó sus ocupaciones de médico y marino para dedicarse a tareas literarias. Sus labores como divulgador científico y ensayista periodístico se completaron con una serie de obras cervantinas, anteriores a la Historia del más famoso escudero Sancho Panza, que reflejan sus ideas y preocupaciones envolviéndolas con el ropaje de su admiración por Cervantes. Se trata de La moral de don Quijote deducida de la Historia que de sus gloriosas hazañas escribió Cide Hamete Benengeli. Por su grande amigo el Cura, impresa por Josef Herrera entre 1789 y 1792; y de La moral del más famoso escudero Sancho Panza, con arreglo a la historia que del más hidalgo manchego don Quijote de la Mancha escribió Cide Hamete Benengeli, publicada, sin nombre de autor, por la imprenta Real en $1793^{6}$.

3. Citaré dentro del texto, señalando parte y página entre paréntesis.

4. Cfr. BArrero PéreZ, Óscar, "Los imitadores y continuadores del Quijote en la novela española del siglo XVIII", Anales Cervantinos, XXIV, 1986, pp. 103-121. Aunque las diferencias entre las dos partes son obvias, como veremos, no es del todo descartable que estas palabras del prólogo no sean sino un disfraz más, característico del tan cervantino juego de autores ficticios, como apunta MANCING, H., "Jacinto María Delgado and Cide Hamete Benengeli: a semi-classic recovered and a Bibliograplical Labyrinth explored", Cervantes, VII, n. ${ }^{\circ}$ 1, 1987, pp. 13-43. Pero en la segunda parte no han quedado huellas que delaten la presencia de Gatell.

5. Vid. Larriba, Elisabel, De la lancette á la pluma. Pedro Pablo Gatell y Carnicer, Aix-en-Provence, Publications de L'Université de Provence, 2005. En esta obra se justifican razonadamente con datos extraídos de periódicos de la época las atribuciones a Gatell de las obras mencionadas y se considera probado no ser de Gatell la Segunda Parte de la novela.

6. En la preliminar "Carta del autor de esta obra a todo el que la leyere" de este último libro hace referencia el autor a su anterior Moral de don Quijote y anuncia también que "ahora pienso, si Dios me diese vida, escribir la Historia del más famoso Sancho Panza desde la gloriosa o envidiable muerte de su amo don Quijote de la Mancha, hasta la última hora de su vida y entierro". 
Son dos obras parecidas donde Gatell habla de la educación, de las clases sociales, de la emigración a América, de la situación del teatro, del elogio del trabajo y de un sinfin de temas más, característicos de la Ilustración, al hilo de una paráfrasis del texto cervantino comentado por Gatell (sin prescindir de referencias que apuntan a hechos autobiográficos) o por Gatell y el cura en el caso de La moral de don Quijote. Y dos obras que, salvando las distancias, pudieran verse como un pálido y desdibujado antecedente de la más brillante Vida de don Quijote y Sancho de Miguel de Unamuno ${ }^{7}$.

Aunque en la carta-prólogo de La moral del más famoso escudero Sancho Panza Gatell avisa que esta obra pretende entresacar la moral de este personaje "sin cuidar ni soñar de imitaciones" del Quijote, lo cierto es que, por las mismas fechas, entusiasmado por la manera en que Cervantes endulza la píldora y enseña deleitando, "animado de todo esto, después que había concluido la Moral de don Quijote y la de su escudero, me propuse escribir un rasgo de su imitación" con la Historia del más famoso escudero Sancho Panza (Primera Parte, sin numerar, folios 2 y 3 ).

Constituye esta obra una verdadera novela que utiliza procedimientos literarios muy distintos de sus obras cervantinas anteriores aunque no desaparecen del todo las pretensiones ensayísticas, los comentarios moralizantes o la propia presencia del autor, siempre disfrazados o entretejidos, claro es, en el desarrollo gradual de la historia, cosa que sucede en menor medida en la Segunda Parte de la novela aparentemente escrita por otro autor.

Los consejos médicos que un profesional experimentado da a un médico novel en la Primera Parte de la novela son así recomendaciones del propio Gatell (Primera Parte, pp. 237 y ss.), y las lamentaciones sobre la degradación del sentimiento amoroso en la época presente corresponden al narrador Gatell:

Un amor sencillo, casto y honesto; no el amor que reina en la época presente; amor interesado, y nada puro el más; amor aparente, que dura solo, que solo se mantiene en el exterior, y cuyas demostraciones son solo hijas del porque no digan. ¡Triste época la presente! (Primera Parte, pp. 35-36).

Más ensayísticas, si cabe, son todavía las páginas correspondientes al capítulo "Particularidades que observó el Bachiller Sansón Carrasco en el tiempo que obtuvo la vara de alcalde el famoso Sancho Panza”. En estas páginas la caracterización de Sancho como prototipo de alcaldes no puede ocultar del todo lo que verdaderamente son: un programa de buen gobierno, cercano a las utopías tan propias del siglo, sobre el orden, la religión y, fundamentalmente, sobre la preocupación esencial de los ilustrados, la educación, cuyos objetivos

7. Cfr. López Navia, Santiago, El autor ficticio Cide Hamete Benengeli y sus variantes y pervivencia en las continuaciones e imitaciones del Quijote, Madrid, Universidad Complutense, 1990; obra revisada como La ficción autorial en El Quijote y sus continuaciones e imitaciones, Madrid, Universidad Europea de Madrid-CEES, 1996. El autor sostiene que el recurso del manuscrito encontrado en La moral de don Quijote, que apunta a la obra cervantina, contiene un juego de narradores que convierten esta obra en una novela y no solo en un ensayo, como parece a simple vista. 
son, no obstante, bien conservadores ya que deben apuntar casi exclusivamente a formar "buenos cristianos, mejores súbditos y ciudadanos útiles" (Primera Parte, p. 283).

Sancho dicta, en efecto, unas "Constituciones" (muy alejadas de aquellas ordenanzas elaboradas por el Sancho cervantino en su gobierno de la Ínsula Barataria) que rijan los fines educativos: los fundamentos son cristianos y éticos pero, obviados estos puntos, el alcalde insiste en dos aspectos esenciales del despotismo ilustrado:

Es necesario que nos instruyan de que entramos en una sociedad; que para ser miembros verdaderos de ella hemos de ser útiles (...) que esta sociedad la gobierna un señor soberano, que conocemos con el nombre de rey; que el mismo Dios le elige y nos manda que le amemos, obedezcamos y sirvamos como a nuestro señor natural, como que en este suelo hace las veces de Dios soberano. (Primera Parte, pp. 288-289).

Se trata además de una educación sexista ("Sancho fundó una escuela para los varones, y otra para las hembras", Primera Parte, p. 281) en la que, frente a las propuestas que por los mismos años lanzaban Montengón o Josefa Amar y Borbón, se reserva a la mujer una formación en "los oficios propios de una mujer de su casa, esto es, coser, hilar, hacer calceta, etc. No se pretende tanto que sean sabias cuanto que salgan buenas mujeres, honradas, hacendosas y enemigas de la ociosidad" (Primera Parte, p. 295) ${ }^{8}$.

El tono general de la obra no es, con todo, ensayístico sino fundamentalmente narrativo, sobre todo en su Segunda Parte, como continuación que es de una obra novelística precedente a la que, como no podía ser de otra manera, está férreamente subordinada.

La Historia del más famoso escudero Sancho Panza, en efecto, apunta una y otra vez a la obra de la que parte: los personajes son los mismos casi en su totalidad, las situaciones son parecidas, los procedimientos narrativos, similares y el recuerdo de episodios del Quijote, constante.

Ya desde las primera páginas se muestra el enlace entre las dos obras al preguntarse, en primera persona, el narrador por qué Cervantes no continuó escribiendo la historia de Sancho. Suponiendo que ello fue debido a que se sintió satisfecho con la muerte del protagonista, Gatell decide proseguirla y, descolgando la pluma a la que se dirigía Cide Hamete, plantear una historia del escudero "sin ser presuntuoso ni malandrín" (Primera Parte, p. 5), como advertía Cervantes a los posibles continuadores en las páginas finales del Quijote.

A partir de este momento todo van a ser recuerdos de la obra cervantina. En su desconsuelo, por ejemplo, Sancho solo se acuerda de las bondades de su amo y le echa tanto de menos que cae gravemente enfermo y en este es-

8. Cfr. Aguilar Piñal, Francisco, "La educación al servicio del progreso en el siglo XVIII", Carlos III y la Ilustración, II, Madrid, Sociedad Económica Matritense, 1990, pp. 45-60. 
tado a menudo delira y, en sueños, se reproducen literalmente pasajes del Quijote:

\begin{abstract}
Algo después de medianoche, entre soñando y despierto daba tales voces, que despierta Teresa se mantuvo quieta escuchando lo que decía para informar al Cura: Señor doctor Pedro Recio de Mal-agüero, natural de Tirteafuera, lugar que está a la mano derecha como vamos de Caraquel a $\mathrm{Al}-$ modóvar del Campo, graduado en Osuna; quíteseme luego de delante, si no, voto al sol, que tome un garrote y que a garrotazos, comenzando por él, no me ha de quedar médico en toda la Ínsula. (Primera Parte, p. 53 y cita textual del capítulo XLVII de la Segunda Parte del Quijote).
\end{abstract}

Una vez recuperado de su melancolía, Sancho encuentra consuelo y recibe admiración de sus vecinos al relatarles "por ápices la historia de su escuderil vida" (Primera Parte, p. 95), tal y como sucede al referirles, después de oír misa, las aventuras de los rebaños de ovejas, casi con las mismas palabras del modelo cervantino (Primera Parte, pp. 98-114), relato que al modo de los comentarios que hacen en el Quijote los oyentes del Curioso impertinente también da pie al trazado de opiniones de los aldeanos; o al contarles la aventura del rebuzno (pp. 152-158).

Este Sancho narrador adereza sus historias con continuas digresiones y, como en el caso del cuento de la pastora Torralva del capítulo XX de la Primera Parte del Quijote, a veces sus relatos dejan de completarse, como sucede con la anunciada aventura del encuentro con el caballero de los Espejos.

Otros episodios son únicamente aludidos, como las referencias a El Curioso impertinente (p. 158, Primera Parte), al discurso armas-letras (p. 148, Primera Parte), a la disputa de don Quijote con el eclesiástico en casa de los duques (p. 76, Primera Parte) y, una y otra vez, a los recuerdos de su etapa como gobernador y los consejos que a este propósito le dio el hidalgo manchego, que sirven de pauta para su actuación como alcalde.

El Sancho de esta Historia rige su comportamiento muchas veces por lo que ha oído decir a su señor o por las experiencias que ha acumulado como escudero. Para organizar un banquete de bodas del que le pide un vecino que se encargue, Sancho tiene presente las famosas bodas de Camacho y las comidas en casa de los duques, de don Diego de Miranda o de don Antonio Moreno, decidiendo al final disponerlo casi todo como en la casa de este último. (Primera Parte, pp. 163 y ss.).

Y, cuando siendo ya alcalde, se presenta en su aldea Ginés de Pasamonte con su retablo y su mono adivino no duda en echarlo del lugar como medio de escarmiento para vagos ("Así se debían castrar tantos zánganos como andan por España comiendo y bebiendo y holgándose con el caudal ajeno", Primera Parte, p. 251) así como recuerda los comentarios de su amo al presentarse en el lugar unos cómicos de la legua el día de la Fiesta de la Cruz, y Sancho solo les deja representar, con criterios muy neoclásicos ahora, obras edificantes.

En la Segunda Parte de la obra la utilización intertextual del Quijote es mucho menos frecuente $\mathrm{y}$, aparte de las alusiones sueltas a la muerte del hi- 
dalgo o a las experiencias de Sancho como gobernador, es más importante la presencia del libro como tal libro que sus propios contenidos: los duques piden a Sansón Carrasco que les cuente los últimos días y la muerte de don Quijote para ver si concuerda con lo que ellos han leído. El Bachiller, además, se sabe personaje de novela al ser reconocido en un mesón como el caballero de la Blanca Luna que venció a don Quijote y le permitió así recobrar el juicio. Y, cuando Sancho recuerda en este mismo mesón la sentencia que emitió cuando era gobernador, un escribano le recrimina:

Amigo, eso son cuentos. Vmd. ha leído en esa nueva Historia de don Quijote de la Mancha y no cuanto allí pone aquel autor morisco es verdad (Segunda Parte, p. 51) ${ }^{9}$.

En esta Segunda Parte, además, Sancho toma conciencia de la realidad de sus vivencias con el hidalgo. Su decepción al no ser recibido por los duques en Madrid y la confesión de un criado de estos le hace ver lo que fue en realidad sus experiencias en la Ínsula ya que "todo cuanto había logrado en su gobierno había sido juego" (Segunda Parte, p. 72) ${ }^{10}$.

Los episodios recreados del referente cervantino tienen que ver, por tanto, con la posibilidad de utilizar materiales narrativos de los que se pueda extraer una enseñanza, útil par organizar una sociedad muy distinta a la cervantina y útil para separar la realidad de la apariencia.

Varios episodios de la Historia del más famoso escudero Sancho Panza son originales de sus autores pero guardan un gran parecido con otros de la obra de Cervantes. El apaleamiento del inocente Sancho en la Primera Parte por parte de unos mozos burlones entre los que se encuentra el bachiller Sansón Carrasco remite al famoso manteamiento del capítulo XVII de la Primera Parte del Quijote. Y la creencia por parte de Sancho de que un gitano le ha robado su asno por ser idéntico al que este monta no puede dejar de asociarse con el famoso robo del asno de Sancho que tantos problemas ha ocasionado a los críticos de todas las épocas. Cuando Sancho reconoce su equivocación, le falta tiempo, además, para relacionar su comportamiento con el característico de don Quijote:

Ahora veo que mi amo no se alucinaba de balde tomando por gigantes los molinos de viento, por castillos las ventas y tantas visiones como tuvo. (Primera Parte, p. 140).

Si los episodios de esta Historia remiten infinidad de veces a la obra de Cervantes, ciertos usos cervantinos en el manejo de la voz del narrador y ciertas

9. Esta misma utilización del Quijote como libro ya se aplicaba en la Primera Parte de la novela, como sucede cuando Teresa advierte a Sancho que sea muy comedido al contar sus recuerdos de don Quijote en presencia del Cura ya que este es sabio y además, como añade Sancho, posee un ejemplar del libro de Cervantes y siempre lo está leyendo.

10. En la Primera Parte ya le había advertido lo mismo Teresa Panza a su marido y este no lo había querido reconocer (p. 117). 
peculiaridades estilísticas suyas son copiadas también por sus autores. La bifurcación de la historia en dos líneas argumentales, por ejemplo, es conducida desde arriba por el narrador con intervenciones en primera persona del plural casi desde el principio de la novela: "Dejemos a Sancho con su mujer y con su hija; y vamos a la tertulia de casa del Cura en aquella misma noche (Primera parte, pp. 42-43); "Veamos ahora qué le sucedió a Sansón mientras que el Cura estaba en casa de Sancho" (Primera parte, p. 79); "Carrasco, apresurado, dijo, agur, agur, y se retiró a su casa, adonde le dejaremos, mientras que pasamos a ver qué hace el alcalde contra Sancho" (Segunda parte, p. 100)11.

Ciertos remates de episodios son genuinamente cervantinos: "Ya había salido el señor párroco para la casa de Sancho, adonde sucedió lo que se verá" (Primera parte, p. 72). Y aunque la novela no está dividida en capítulos numerados, algunos de los títulos que encabeza cada una de las secciones, arbitrariamente establecidas muchas veces, recuerdan las humorísticas entradas del Quijote: "De la impensada aventura que tuvo Sancho Panza en la mañana siguiente de la apaleadura de los mozos" (Primera parte, p. 205); "Historia de la sonada aventura de Sancho, en donde verá y leerá el lector cosas nunca oídas" (Primera parte, p. 205); "Observación digna de la más escrupulosa atención" (Primera parte, p. 298) ${ }^{12}$.

La estructura de la Historia del más famoso escudero Sancho Panza debe mucho también al Quijote. La distribución de los sucesos narrativos en dos partes y las peculiaridades de cada una de ellas recuerdan, a lo lejos, a la obra de Cervantes. En la Primera Parte, un largo comienzo preparatorio de la acción nos presenta a un Sancho desconsolado e inapetente que hace temer a su mujer "llegase a perder el juicio" (Primera parte, p. 13), exactamente igual que el hidalgo de las primeras páginas del Quijote, bien que por motivos distintos. Y, como en la obra de Cervantes, los personajes de la aldea, el cura, el barbero y el Bachiller Sansón Carrasco, pretenden sacarle de su tristeza y de los atisbos de locura que experimenta.

El final de la novela es idéntico al del Quijote: un Sancho enormemente entristecido por el comportamiento miserable del nuevo alcalde de su aldea, que le ha encarcelado y le ha acusado injustamente de contrabando, muere melancólico y muy cristianamente no sin antes renegar de las aventuras con su lunático amo:

Mi aflicción de unos días a esta parte, es cúan tarde conocí el desatino de dejar mi casa, abandonar mi familia por seguir las locuras de mi amo. (Segunda Parte, pp. 256-257).

11. Aunque la novela está escrita en tercera persona, a veces el narrador a la manera cervantina interrumpe la acción para quejarse, en primera persona, de la falta de noticias en su información, como cuando, con ocasión de la venida de los duques a la aldea, lamenta desconocer el contenido de la conversación que aquellos mantienen con Sancho: "Lo que hablaron allá dentro no ha podido llegar a mi noticia; por eso no lo pongo aquí, con harto dolor de mi ánima" (Primera parte, p. 267).

12. Los títulos de la Segunda parte, en cambio, son, más bien, un resumen argumental de unos capítulos que ya tienen mayor unidad narrativa. 
Y entre el principio y el final una estructura circular de viaje, al menos en la Segunda Parte, que es una copia de la característica estructura quijotesca: planteamiento de la acción, viaje y aventuras y vuelta a casa.

En cambio no aprovechó el autor la división en capítulos ya que estos en general no tienen unidad narrativa.

Como en el Quijote, la Historia del más famoso escudero Sancho Panza incluye cartas, discursos e infinidad de diálogos... que carecen de la ligereza y agilidad de los típicamente cervantinos.

Pero donde los autores de esta novela no podían sino seguir a Cervantes es, si no en el arte de la caracterización, sí en la nómina de protagonistas. Muy pocos son, en efecto, los personajes nuevos y carecen casi todos de importancia ya que o son simplemente anecdóticos, como la Tía Paca de la Primera Parte, cotilla oficial de la aldea que divulga la inicial inquina que siente Sancho por el Bachiller Sansón Carrasco, a quien culpa de la muerte del hidalgo; o bien sirven de contrapeso para poner de manifiesto las bondades del escudero, como en el caso del labrador que ruega a Sancho, como hombre experimentado, que le organice la boda de su hijo, o los casos del tío Cosme o del nuevo alcalde de la aldea, que permiten mostrar la honradez y generosidad de Sancho al enfrentarle a un inocente y a un miserable respectivamente.

Estos personajes secundarios apenas si están esbozados y hasta carecen muchas veces de nombre propio. Pero incluso en los tomados de Cervantes no es mucho mejor el arte de la caracterización, tan alejada de la proverbial maestría del escritor alcalaíno. El cura es simplemente un depositario de la prudencia y el consejo, y participa mínimamente en la acción. Menos lo hace todavía el barbero. Y el bachiller Sansón Carrasco es un personaje apagado que, aunque se va apoderando gradualmente del protagonismo en la Segunda Parte de la novela y aunque es decisivo con sus manejos y astucias para demostrar la inocencia de Sancho, carece de la socarronería y el humor del original por mucho que los autores intenten poner de manifiesto sus burlas y maldades, a las que se les da la vuelta al final de la historia para que sirvan de muestra de las bondades de Sancho.

Los duques de la segunda parte de El Quijote pasan como sombras por la novela, testigos únicamente de los cambios habidos en el carácter de Sancho, garantes de la seguridad económica de la familia Panza tras la muerte de Sancho y excusa para que este emprenda un viaje a la Corte y se desengañe de sus aparentemente generosos protectores en una nueva formulación del tópico del menosprecio de corte y alabanza de aldea.

Algo mejor dibujada está la figura de Teresa Panza en cuya caracterización, pero no sin contradicciones, algo avanza esta novela frente al original del que parte: amante esposa, ayuda y aconseja a Sancho en todo momento y transmite a la novela una visión del mundo muy conservadora basada en el elogio del trabajo manual, en la gramática parda y en la unidad familiar. Sin embargo, en la Segunda Parte, es la incitadora y motor primero de la desgracia final de Sancho al recriminarle su poca habilidad para sacar provecho del 
cargo de alcalde que ha ocupado y al obligarle en última instancia a acudir a Madrid para solicitar favores a los duques.

Pero donde más claramente se comprueba la distancia entre el original y su continuación es en el tratamiento del protagonista. En su benemérito Catálogo don Gabriel Martín del Río y Rico indicaba que "el carácter de Sancho está bien dibujado y sostenido, y se parece mucho al modelo"13. Posiblemente no sea para tanto. La gracia de su lenguaje ha desaparecido casi por completo, apenas si utiliza los refranes tan jugosos del Quijote y más que inocente hombre de bien lleno de picardía que se va puliendo al contacto con el hidalgo, aquí se nos muestra como un sabio, honrado hasta la exageración, insobornable, generoso, "discursivo, con posibilidad de distinguir y aconsejar" (Segunda Parte, prólogo sin numerar), astuto y hábil administrador, ilustrado alcalde que no sabe leer ni escribir.

Algunos de estos rasgos ya estaban en potencia en el original pero los recovecos de su carácter y la verdad humana del personaje cervantino se han esfumado para dar paso a un personaje de una pieza que además va perdiendo protagonismo conforme avanza la novela ${ }^{14}$. Quizás esta simplificación psicológica no sea sino el tributo que debe pagar el autor para amoldar un personaje creado por Cervantes en circunstancias históricas muy diferentes al pensamiento ilustrado, que dibujaba un ideal humano (el hombre de bien) de honradez, discreción y trabajo, de utilidad y autocontrol de que este Sancho pudiera ser representante: 15

Considerándose un padre de todos los vecinos de la aldea, se desvelaba por mantener el buen orden en todas las cosas: amonestaba a unos y consolaba a otros; y cual buen pastor y padre castigaba a quien no quería atender a sus silbidos (Primera Parte, p. 282) ${ }^{16}$.

13. Vid. Río y Rico, Gabriel Martín, Catálogo bibliográfico de la sección Cervantes de la Biblioteca Nacional, Madrid, 1930, p. 586.

14. Las diferencias entre el Sancho del Quijote y el de la Historia la advierten los propios personajes. Al comienzo de la novela, dice el Cura: "Enseñado ya por el ejemplo de su amo, ilustrado con sus conversaciones y ayudado de algunas luces naturales, es muy otro Sancho de lo que podía pensarse de un hombre tosco y sin letras" (Primera Parte, p. 46).

15. Muchas de las virtudes que se catalogan en los Ensayos de filosofía moral o El hombre de bien, traducidos al español por Agustín de Arcos (religiosidad, rectitud, justicia, liberalidad, humanidad, amor a la patria, etc.) están muy presentes en el carácter de este Sancho. Cfr. ÁlvAREZ BARRIENTOS, Joaquín, Ilustración y Neoclasicismo en las letras españolas, Madrid, Síntesis, 2005, pp. 113 y ss.

16. Cuando en la Segunda Parte Sancho hace frente a las insinuaciones de su mujer acerca de que no ha sabido aprovecharse del cargo de alcalde, le dice que "la honra y la voz común importan más que cuantos intereses puedan adquirirse" y que él está contento de tener la "conciencia limpia" (Segunda Parte, pp. 8 y 10). En cambio, otro rasgo del carácter de Sancho en esta novela, el de la melancolía, es más discutible que tenga que ver con rasgos ilustrados ya que no se presenta en su persona ese "fastidio universal" del que hablara Meléndez Valdés en una oda famosa. Las causas de la melancolía de Sancho son muy concretas: muerte de don Quijote y sentimiento de desamparo al comienzo de la novela; decepción por la actitud de los duques y pena por ser encarcelado injustamente al final de la obra. 
Y si la Historia del más famoso escudero Sancho Panza no puede sostener la hondura en la caracterización de Cervantes, menos aún puede mantener otros rasgos definitorios de su arte de novelar: la ironía ha desaparecido y no hay ni asomos del juego de perspectivas que tanto han admirado escritores de todos los tiempos. Ni siquiera el humor se conserva.

En las "Prevenciones al que leyere esta obra", Gatell se siente obligado a advertir sobre el carácter luctuoso del comienzo de la novela, y el presumible segundo autor de la Historia marca distancias, precisamente, con las Adiciones de Delgado por ser su obra seria y no solamente burlesca, como esta:

La idea del primer tomo es seria, y su formación va coordinada, y separada totalmente de la ficción burlesca, y que camina al conocimiento de un hombre, que algo ilustrado con la experiencia, advertía el error de haber seguido los desatinos tan crasos de un caballero andante como su amo don Quijote, de suerte que no ligan bien las referidas adiciones con el primer tomo, porque en ellas siguen las burlas y en este pensamiento no" (Segunda Parte, prólogo sin numerar, folio 5).

El programa se sigue a rajatabla y el humor casi desaparece por completo. Incluso cuando relata algo chistoso, como la caída de su cama matrimonial tras una noche de intranquilidad y vanidad luego de ser nombrado alcalde, lo hace sin demasiada gracia y pasando como por ascuas por un motivo del que Cervantes hubiera sacado mucho partido. (Primera Parte, pp. 223-224).

Es mucho, por tanto, lo que toma la Historia del más famoso escudero Sancho Panza del Quijote y mucho más lo que deja de tomar. Su aportación original, además de una nueva visión del mundo y un cierto tono ensayístico, que desaparece casi por completo en la Segunda Parte, reside en su argumento, narrado linealmente y que puede resumirse con brevedad:

Primera Parte: Después de una grave enfermedad ocasionada por la muerte de don Quijote, Sancho empieza a adquirir notoriedad en su aldea por los relatos de sus aventuras con el hidalgo y por su discreción y capacidad de aconsejar sabiamente. Por esto mismo, el Ayuntamiento le nombra su apoderado para representarle en un litigio, del que sale con éxito, y sus convecinos le eligen como alcalde. Durante su mandato, marcado por la honradez y la sabiduría, se solucionan con justicia todos los conflictos y se establece un programa de reformas del que todos se encuentran tan satisfechos que quieren que continúe en ese cargo. Pero Sancho lo rechaza así como rechaza la propuesta de los duques, de paso por la aldea, de ser de nuevo gobernador.

Segunda Parte: Un Sancho labrador casi arruinado que no se ha aprovechado en ningún sentido de su cargo de alcalde, es instado por su mujer para que solicite favores de los duques en Madrid. Pese a su oposición inicial, Sancho acaba acudiendo a la corte, pero no logra ser recibido por los duques y obtiene escaso rendimiento a su viaje. A la vuelta a la aldea, un nuevo alcalde rencoroso decide perjudicar a Sancho y, tras ocultar maliciosamente en la casa del escudero mercancías variadas, le acusa de contrabandista y le lleva a la cárcel. El buen hacer del Bachiller Sansón Carrasco y sus maquinaciones con 
los duques consiguen esclarecer la verdad y sacar a Sancho de la cárcel. Pero este padece un acceso de melancolía que le lleva a la muerte.

Como se avisa en el prólogo de la Segunda Parte, "pensar que a la elocuencia y maestría de Cervantes puede llegar alguno en el día es pedir peras al olmo" (Segunda Parte, prólogo, sin numerar, folio 3). Los autores de la Historia del más famoso escudero Sancho Panza se quedaron muy lejos del original, ciertamente, pero su novela es un homenaje verdadero a Cervantes y una manera de atestiguar que, siguiendo a Cervantes, era posible, a finales del siglo XVIII, crear una forma literaria en la que el entretenimiento y el interés por la historia, y no solo la enseñanza y el didactismo, fueran la clave ${ }^{17}$.

\title{
Resumen
}

La Historia del más famoso escudero Sancho Panza es una de las más características continuaciones del Quijote del siglo XVIII. Tras situar la obra en su contexto, el artículo traza un estudio comparativo (coincidencias episódicas, en personajes, estructura y estilo; y diferencias sustanciales entre las dos novelas) para mostrar el alcance de la consideración cervantina durante esta época.

Palabras clave: Gatell. Siglo XVIII. Literatura comparada. Intertextualidad. Tradición cervantina. Sancho Panza. Cervantes.

\begin{abstract}
The Historia del más famoso escudero Sancho Panza is one of the most caracteristic continuation of the Quijote in the XVIIIth siecle. After situating the play in its context, this article studies comparatively both novels to show the influence and importance of Cervantes during this époque.
\end{abstract}

Key words: Gatell. 18th Century Literature. Comparative Literature. Intertextuality. Cervantine tradition. Sancho Panza. Cervantes.

17. Sobre el significado del quijotismo en el siglo XVIII, además de los estudios ya citados, Cfr. Álvarez BARrientos, Joaquín, La novela del siglo XVIII, Madrid, Júcar, 1991; y García LARA, Fernando, "Condición hipertextual de la novela del siglo XVIII", El siglo que llaman ilustrado. Homenaje a Francisco Aguilar Piñal, Madrid, CSIC, 1996, pp. 399-405. 